\title{
Damage Localization, Sensitivity of Energy Release and the Catastrophe Transition
}

\author{
Hui Ling Li, ${ }^{1}$ Zhao Ke Jia, ${ }^{1}$ Yi Long Bai, ${ }^{1}$ \\ Meng Fen XIA, ${ }^{1,2}$ and Fu JiU Ke ${ }^{1,3}$
}

\begin{abstract}
Large earthquakes can be viewed as catastrophic ruptures in the earth's crust. There are two common features prior to the catastrophe transition in heterogeneous media. One is damage localization and the other is critical sensitivity; both of which are related to a cascade of damage coalescence. In this paper, in an attempt to reveal the physics underlying the catastrophe transition, analytic analysis based on mean-field approximation of a heterogeneous medium as well as numerical simulations using a network model are presented. Both the emergence of damage localization and the sensitivity of energy release are examined to explore the inherent statistical precursors prior to the eventual catastrophic rupture. Emergence of damage localization, as predicted by the mean-field analysis, is consistent with observations of the evolution of damage patterns. It is confirmed that precursors can be extracted from the time-series of energy release according to its sensitivity to increasing crustal stress. As a major result, present research indicates that the catastrophe transition and the critical point hypothesis $(\mathrm{CPH})$ of earthquakes are interrelated. The results suggest there may be two cross-checking precursors of large earthquakes: damage localization and critical sensitivity.
\end{abstract}

Key words: Damage localization, sensitivity of energy release, heterogeneous medium, catastrophic rupture.

\section{Introduction}

Large earthquakes can be viewed as catastrophic ruptures in the earth's crust. There is evidence that complex spatial and temporal patterns of seismicity develop prior to the occurrence of a main rupture. In fact, in addition to event clusters in the vicinity of the nucleation zone of a mainshock, apparent acceleration of seismic activity of moderate-sized earthquakes over a large area (SAMmis et al., 1994; JAUMÉ and SYKES, 1999; RUNDLE et al., 2000; XIA et al., 1997) has been observed. From the viewpoint of

\footnotetext{
${ }^{1}$ State Key Laboratory of Nonlinear Mechanics, Institute of Mechanics, Chinese Academy of Sciences, Beijing 100080, China.

E-mails: lihl@Inm.imech.ac.cn, jiazk@1nm.imech.ac.cn, baiyl@lnm.imech.ac.cn

${ }^{2}$ Department of Physics, Peking University, Beijing 100871, China.

E-mail: xiam@lnm.imech.ac.cn

${ }^{3}$ Department of Applied Physics, Beijing University of Aeronautics and Astronautics, Beijing 100083, China. E-mail: kefj@lnm.imech.ac.cn
} 
rock mechanics, fracture surface formation and accelerated release of stored elastic energy may be two essential aspects underlying the observed seismicity patterns. Earthquakes in the crust, like all other ruptures which occur in heterogeneous media, result from an unstable cascade of damage coalescence beyond a critical transition - a sequential process of progressive microcracking of defects owing to nonlinearity of evolution and heterogeneity in microstructures. Fracture surface formation is preceded by a process of damage localization in heterogeneous media, while the release of stored energy corresponding to microcracking may imply that precursory activity precedes that main rupture. Consequently, damage localization and sensitivity related to energy release may be two cross-checking precursors of the catastrophe transition. Understanding damage localization and sensitivity of energy release before catastrophe transition may provide deep insight into the physics underlying earthquake ruptures, and thus shed new light on the prediction of a main rupture.

In view of the fact that faults in nature are generally heterogeneous in both size and strength, it is essential to include effects of heterogeneity into consideration in order to study the nature of earthquake rupture. Moreover, stress redistribution caused by microcracking and interactions between faults results in an incredibly complex process of nonlinear dynamics distant from equilibrium. The heterogeneous and nonlinear nature underlying such a complex process suggests that formalism from statistical physics is necessary. Collective behavior of distributed microcracks and microvoids in a heterogeneous medium has been investigated from the viewpoint of statistical mesoscopic damage mechanics in an attempt to establish a trans-scale correlation between such microstructural effects and eventually macroscopic catastrophic fracture (BAI et al., 1998). It can be inferred, from both theoretical analysis and numerical simulations, that macroscopically localized damage serves as an effective precursor of brittle rupture (see BAI et al., 2000a). A criterion for damage localization may allow us to judge the impending rupture in heterogeneous media from the extent to which microfractures cluster together. Meanwhile, sensitivity of energy release characterizes the temporal pattern prior to earthquake rupture.

In this paper, a statistical model based on the mean-field approximation is presented to introduce the criterion for occurrence of damage localization, and examine the sensitivity of energy release. The catastrophe transition which occurs in a damaged heterogeneous medium loaded by surrounding crust is investigated as a possible mechanism underlying the eventual rupture. As a more realistic approximation to the rupture process of fault systems, numerical simulations based on a network model are carried out in an attempt to reveal the complex nonlinear dynamics underlying the process. The heterogeneity in microstructural strength is described by a statistical distribution, and the effect of such distribution on the behavior in the vicinity of eventual rupture is emphasized. Both the emergence of damage localization and the sensitivity of energy release are examined in search of the inherent statistical precursors prior to catastrophic rupture. It is confirmed that precursors can be extracted from the data of energy release according to its sensitivity to the increasing stress. Emergence of 
damage localization can be predicted by a criterion which is consistent with observations of the evolution of damage patterns. It may be concluded that damage localization and sensitivity of energy release characterize fracture surface formation, and thus provide two cross-checking precursors for the prediction of rupture.

\section{Mean-field Approximation in a Statistical Model}

Here, the statistical physics underlying the spatial, temporal and magnitude distribution of events have been investigated, based on a statistical mean-field model to a first approximation (KrajCINOvic, 1996; XIA et al., 1996). We consider a body which consists of a number of linear, elastic bonds. Under macroscopic loading, each bond is stretched and then breaks when its tensional strain exceeds a threshold. The threshold is referred to as the mesostrength while the stress to break the whole body is the macroscopic strength. The heterogeneity of materials may be represented by a stochastic distribution of mesostrength. In fact, simulations based on the statistical ensemble evolution of a dynamical system, consisting of bundles of fiber with a Weibull distribution of mesostrength, explained the diversification of macroscopic strength of heterogeneous materials very well (see BAI et al., 2000b). Thus, to resemble heterogeneous fault systems, the mesostrength of bonds is randomly assigned according to a Weibull distribution

$$
\varphi\left(\sigma_{c}\right)=m\left(\frac{\sigma_{c}^{m-1}}{\eta^{m}}\right) \exp \left[-\left(\frac{\sigma_{c}}{\eta}\right)^{m}\right],
$$

where $\sigma_{c}$ represents the breaking threshold of each bond. Then, $\varphi\left(\sigma_{c}\right) \mathrm{d} \sigma_{c}$ represents the probability a given bond has a strength between $\sigma_{c}$ and $\sigma_{c}+\mathrm{d} \sigma_{c}$. In the following we will adopt that $\eta=1$, thus strength and stress are in units of $\eta$. The Young's modulus $K_{0}$ is assumed to be identical for all bonds. By taking $K_{0}=1$, the real stress is represented by the rescaled strain $\varepsilon\left(\varepsilon=\operatorname{strain} \cdot K_{0} / \eta\right)$. To a first approximation, the strain $\varepsilon$ is roughly assumed to take a uniform value over the whole body. Subsequently the damage variable, defined as the fraction of the bonds which are broken, can be expressed as

$$
D(\varepsilon)=\int_{0}^{\varepsilon} \varphi\left(\sigma_{c}\right) \mathrm{d} \sigma_{c} .
$$

The nominal stress $\sigma$ on the body is related to the strain $\varepsilon$ (the real stress) by the following relationship in damage mechanics

$$
\sigma(\varepsilon)=(1-D(\varepsilon)) \cdot \varepsilon=\left(1-\int_{0}^{\varepsilon} \varphi\left(\sigma_{c}\right) \mathrm{d} \sigma_{c}\right) \cdot \varepsilon .
$$

Corresponding stress-strain curves are shown in Figure (1). 


\section{Criterion for Damage Localization}

From the viewpoint of statistical mesoscopic damage mechanics, a quantitative evolution equation governing the collective behavior of microdamage has been established, based on a statistical treatment. It is inferred that macroscopically localized damage serves as a precursor of eventual rupture, and a criterion for damage localization in heterogeneous damaged material was proposed, on the basis of the statistical evolution equation mentioned above. A detailed derivation is given in BAI et al. (1998) or Li et al. (2000) for a general case. Here we cite the main result and show its application to the simple case described above.

In order to examine damage localization, we divide the system into small macroscopic elements, and apply the mean-field approximation to each element. This is called the local mean-field approximation. As a criterion for the emergence of damage localization, the relative gradient of damage begins to increase with time, i.e.,

$$
\frac{\partial}{\partial t}\left[\left(\frac{\partial D}{\partial z}\right) / D\right] \geq 0,
$$

where $z$ is the spatial coordinate of macroscopic elements. Under quasi-static onedimensional small deformation, the criterion can be reduced to

$$
\frac{\partial \dot{D}}{\partial D} \geq \frac{\dot{D}}{D},
$$

where $\dot{D}=\frac{\partial D}{\partial t}$, provided $\frac{\partial D}{\partial z}>0$. Applying Equation (2) and Equation (3) to each macroscopic element, Equation (5) gives

$$
D(1-D) \varphi^{\prime} \sigma \geq(1-D)^{2}(1-2 D) \varphi-\varphi^{2} \sigma
$$

where $\varphi^{\prime}(\varepsilon)=\mathrm{d} \varphi / \mathrm{d} \varepsilon$. For the Weibull distribution of strength given by Equation (1), the alarm given by Equation (6) is shown in Figure 1 in comparison with the whole stress strain curve (thin line, $\varepsilon_{D L}$ ).

\section{Sensitivity of Energy Release}

An earthquake may be viewed as a catastrophe transition to the main rupture in the heterogeneous crust. From the viewpoint of damage mechanics, the cascade of damage coalescence is the underlying mechanism and causes informative fluctuations ahead of the transition, as pointed out by LU et al. (1999), based on a twodimensional evolution induced catastrophe model. Accordingly, we examine the variation of the energy release rate, or more specifically, the sensitivity to perturbations in the external load (XIA et al., 2000). In the model, even a minor increment in external load may cause a significant response in energy release, as the damaged body approaches the catastrophe transition. Generally, the sensitivity $S$ is defined as 


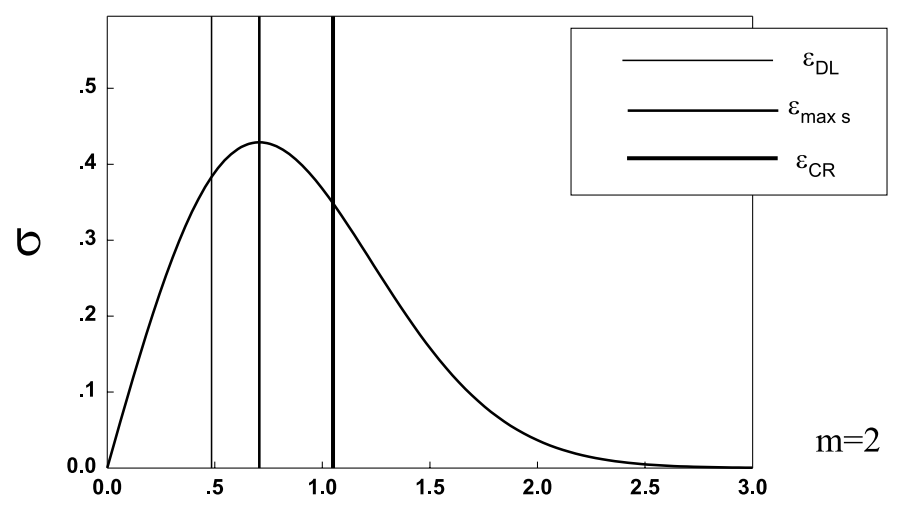

a)

$\varepsilon$

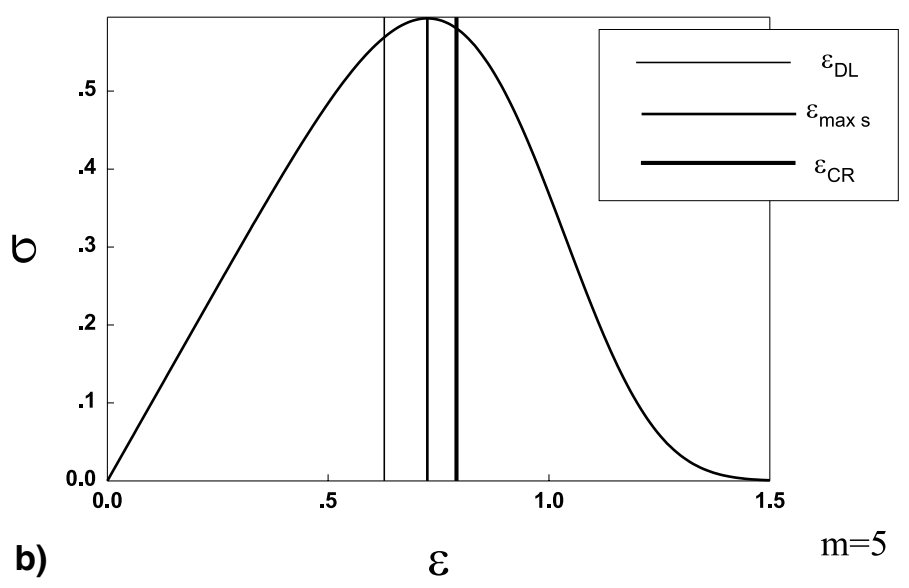

Figure 1

The stress-strain relation in a mean-field approximation of a heterogeneous model possessing a Weibull distribution function with (a) $m=2$; (b) $m=5$. The three vertical lines (from left to right) indicate damage localization $\left(\varepsilon_{D L}\right)$, maximum stress $\left(\varepsilon_{\max s}\right)$ and catastrophic rupture $\left(\varepsilon_{C R}\right)$ successively $(k=0.4)$.

$$
S=\frac{\Delta E^{\prime} / \Delta \sigma^{\prime}}{\Delta E / \Delta \sigma},
$$

where $\Delta E$ and $\Delta E^{\prime}$ are energy release induced by increments of stress $\Delta \sigma$ and $\Delta \sigma^{\prime}$ respectively, and $\left(\Delta \sigma^{\prime}>\Delta \sigma\right)$.

Now we return to the previous model as shown in Figure 1. The work done by the external force can be estimated to be $V \int_{0}^{\varepsilon} \sigma \mathrm{d} \varepsilon$, where $V$ is the total volume of the body. For the damaged brittle medium, the unloading process is assumed to be linear elastic until both stress and strain become zero. Thus the stored elastic energy (strain energy) is $(1 / 2) \sigma \varepsilon \cdot V$. In reality, a fraction of energy may be released to form fault gouge or seismic radiation. Thus, the cumulative energy release can be defined as 


$$
E=\int_{0}^{\varepsilon} \sigma \mathrm{d} \varepsilon-\frac{1}{2} \sigma \varepsilon
$$

When the damage evolution process is controlled by the nominal stress, the release of energy is described by

$$
\frac{\mathrm{d} E}{\mathrm{~d} \sigma}=\frac{\mathrm{d} E}{\mathrm{~d} \varepsilon} / \frac{\mathrm{d} \sigma}{\mathrm{d} \varepsilon}=\frac{1}{2} \cdot \frac{\varphi(\varepsilon) \cdot \varepsilon^{2}}{1-\varphi(\varepsilon) \cdot \varepsilon-\int_{0}^{\varepsilon} \varphi\left(\sigma_{c}\right) \mathrm{d} \sigma_{c}} .
$$

In this case, the mean-field approximation with continuous distribution $\varphi$ provides a simple expression for the sensitivity. We expand $\Delta E$ and $\Delta E^{\prime}$ in terms of finite $\Delta \sigma$ and $\Delta \sigma^{\prime}$ to the lowest order, then

$$
S=\left(\frac{\mathrm{d}^{2} E}{\mathrm{~d} \sigma^{2}} / \frac{\mathrm{d} E}{\mathrm{~d} \sigma}\right) \cdot \frac{\Delta \sigma^{\prime}-\Delta \sigma}{2}+1 .
$$

Without loss of generality, presume $\left(\Delta \sigma^{\prime}-\Delta \sigma\right)$ to be a fixed value, then the sensitivity $S$, depends only on $R=\left(\left(\mathrm{d}^{2} E / \mathrm{d} \sigma^{2}\right) /(\mathrm{d} E / \mathrm{d} \sigma)\right)$, i.e., $S \propto\left(\left(\mathrm{d}^{2} E / \mathrm{d} \sigma^{2}\right) /(\mathrm{d} E / \mathrm{d} \sigma)\right)$. As indicated by the value of $R$ in Figure 2, $S$ increases rather dramatically before the catastrophe transition becomes possible. Such a feature is called critical sensitivity (XIA et al., 2000).

\section{Catastrophe Transition Involving Elastic Surroundings}

Earthquakes usually occur in a seismic area loaded by surrounding crust. For a body loaded from its surroundings, the catastrophe transition manifests itself when

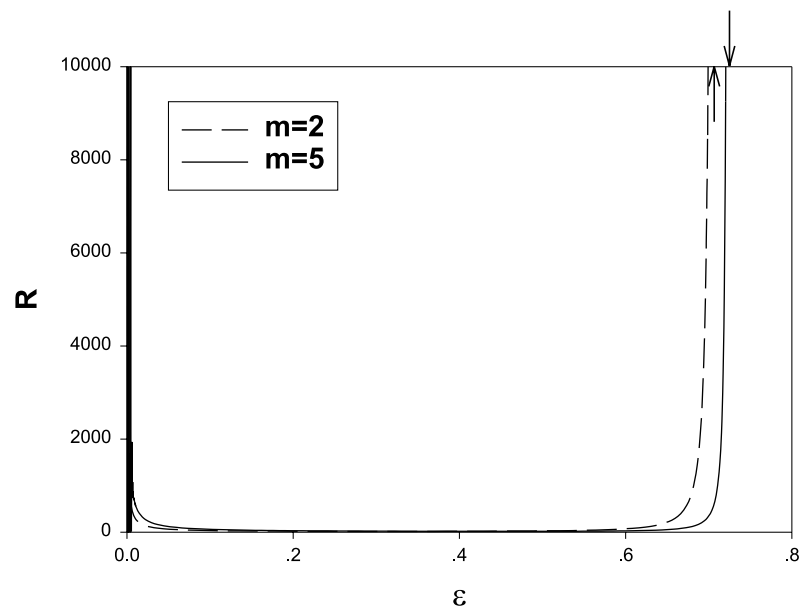

Figure 2

$R=\left(\left(\mathrm{d}^{2} E / \mathrm{d} \sigma^{2}\right) /(\mathrm{d} E / \mathrm{d} \sigma)\right)$ versus stress for a Weibull distribution, indicating the accelerated increase in the sensitivity of energy release to external stress as catastrophic rupture approaches. The arrows ( $\uparrow$ for $m=2, \downarrow$ for $m=5$ ) indicate the time of maximum stress appearance. 
the energy suddenly released by the surroundings exceeds the capacity absorbable by the body (JAEGER and COOK, 1979), i.e.,

$$
\Delta W=F \cdot \Delta u=F \cdot\left(\Delta u_{b}+\Delta u_{s}\right)=F \cdot \Delta F \cdot\left(\frac{1}{K_{b}}+\frac{1}{K_{s}}\right)<0,
$$

where $F$ is force and $u$ is displacement, $u_{b}, K_{b}$ and $u_{s}$ and $K_{s}$ are the displacements and the current stiffness of the body and its surrounding, respectively. Thus the superfluous part of energy may serve to impel the abrupt rupture of the body. If the force $F$ and the surrounding's stiffness $K_{S}$ are always positive, the body would become unstable when

$$
K_{b}<-K_{s} \text { provided } \Delta F<0 \text { and } K_{b}<0 .
$$

Therefore, the catastrophe transition of the body would occur somewhere beyond or at the point of maximum load, when $K_{s}>0$ or $K_{s}=0$, in the diagram of $F$ versus $u_{b}$.

For the mean-field model, inequality (9) can be rewritten as

$$
-\frac{\mathrm{d} \sigma}{\mathrm{d} \varepsilon}>k
$$

where $k=K_{s} / K_{b}$. Critical values for damage variable $D_{c R}$ and strain $\varepsilon_{c R}$ are then obtained (see Figure 1).

Finally, we compare the predictions given by the criterion for damage localization (Equation 6), the condition for maximum stress and the catastrophe transition (Equation 10) in Figure 1. The figures clearly demonstrate that damage localization (Equation 6), maximum stress (corresponding to $K_{s}=0$ in Equation 10) and catastrophic rupture occur successively with increasing deformation. It can be shown that the emergence of damage localization occurs prior to the maximum stress, while the catastrophe transition point tends toward the maximum stress as $K_{s} \rightarrow 0$.

\section{Numerical Simulation of Catastrophic Rupture}

We further examine damage localization and critical sensitivity of energy release on a more realistic basis, by means of numerical simulations. Here we resort to a computational approximation of the rupture process which includes effects of heterogeneous microstructures and the nonlinear dynamics of stress redistribution in heterogeneous media like fault systems.

\section{A Network Model}

The simulations were performed on a two-dimensional network model developed in LIU and LIANG (1998). It consists of a regular triangle lattice of elastic bars. The bars are stretched or contracted linear-elastically. Elastic equations of the system are expressed in terms of a load vector which is related to the displacement of nodes by a stiffness matrix. Solving the set of linear-elastic equations produces the behavior of the lattice subjected to increasing displacement (or loading) at the boundary. 


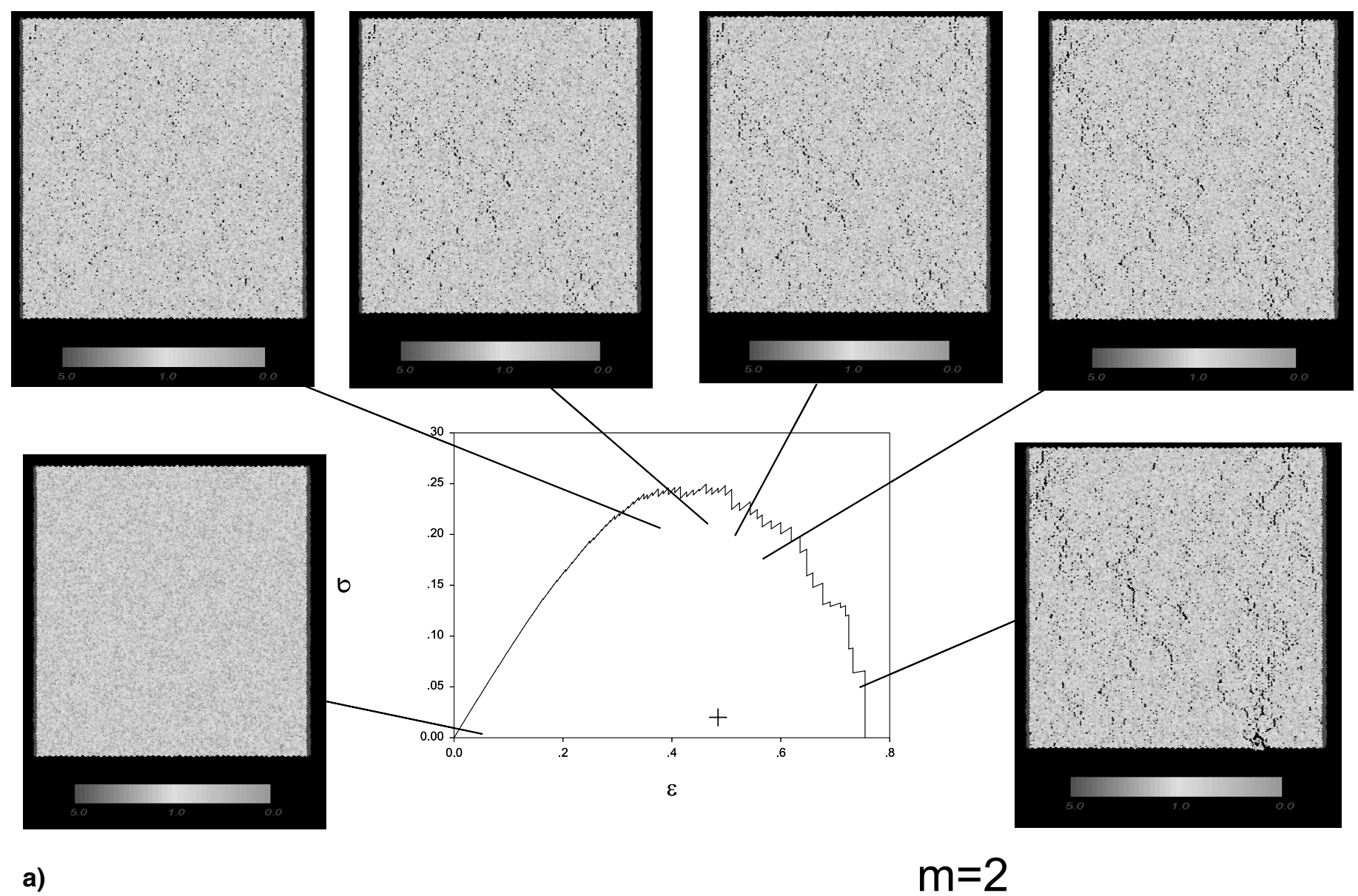

The simulated stress-strain relation of a heterogeneous model possessing a Weibull distribution function with (a) $m=2$ (b) $m=5$ and corresponding damage patterns. The cross $(+)$ indicates the damage localization condition (Equation 6). 


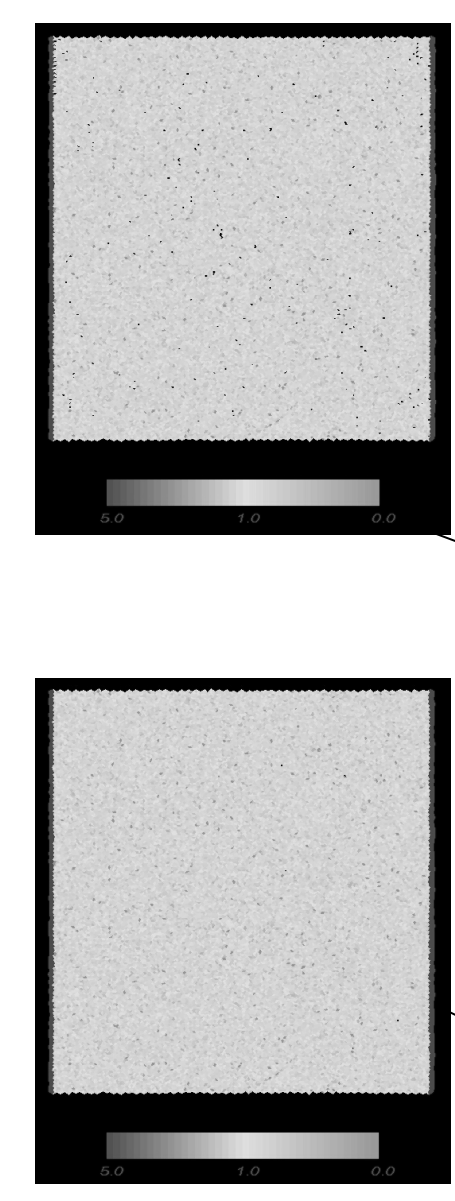

b)

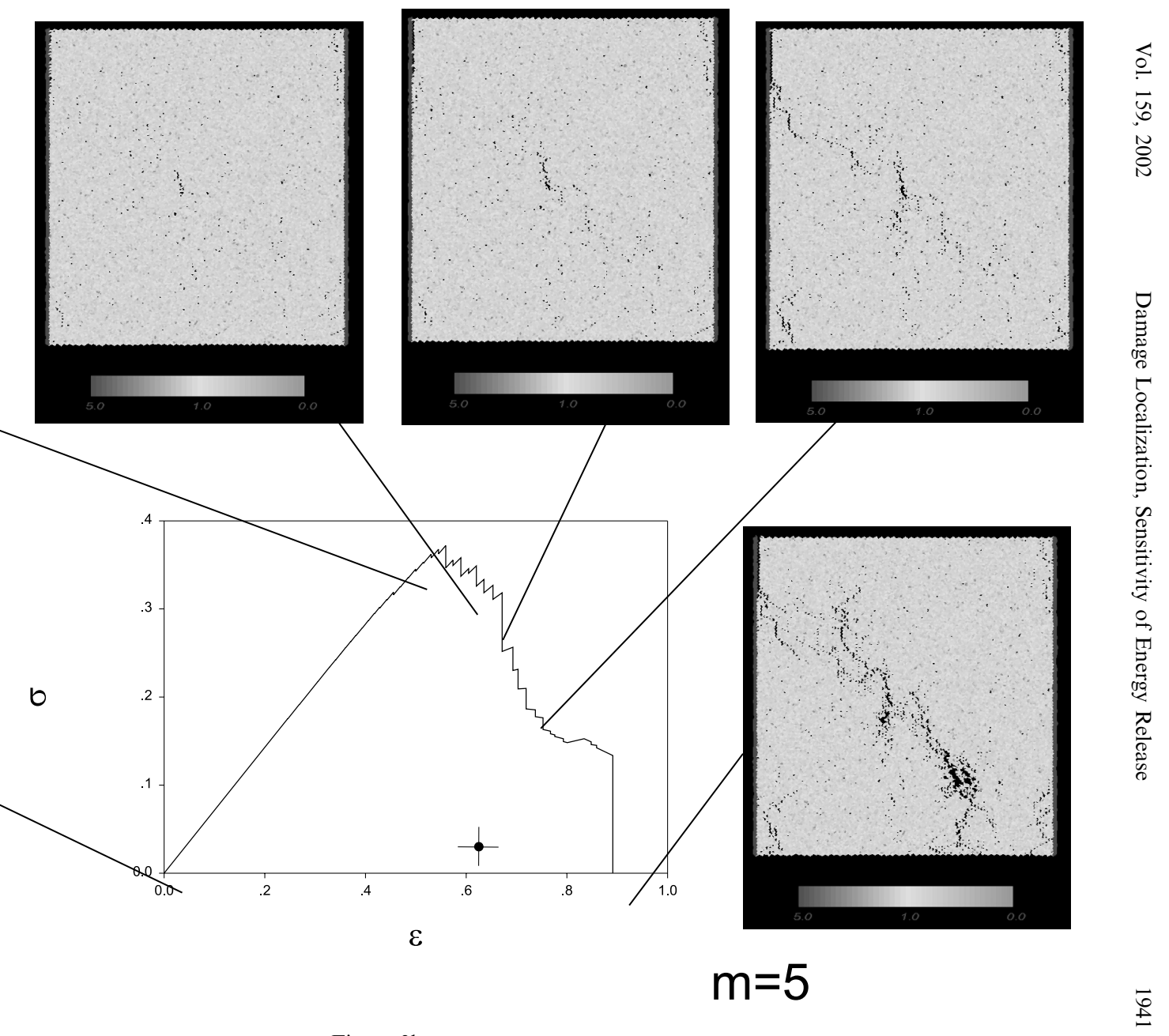

Figure 3b 
To simulate the progressive microcracking process and the sequential rupture in the medium, a breaking rule must be defined for the bars. In the present model, a bar breaks when its stretching strain exceeds a randomly predefined threshold. Without loss of generality, it is assumed that a Weibull distribution as given in Equation (1) describes the threshold properly. In this way the network model resembles a heterogeneous fault system.

Each time the loading or displacement at boundary is increased, the set of elastic equations is solved to obtain the displacement of all nodes in the lattice. Then we check the tensional strain of each bar. When its threshold is exceeded, a bar is broken and removed from the lattice. Once all broken bars have been removed from the lattice, the set of equations is solved repeatedly to permit stress redistribution, and the resultant breakage of bars. Such iterations continue until the tensional strain of all unbroken bars is below their thresholds. When there is no further bond breakage, an equilibrium state (under the present boundary conditions) is obtained. Then the boundary displacement or loading is incremented and the entire procedure iterates. According to this procedure, the spatial evolution of damage patterns and stressstrain curves is obtained (see Figure 3). We employ these results to investigate the physics underlying catastrophic rupture.

\section{Numerical Simulation and Discussions}

An example of deformation and damage patterns in the heterogeneous model under strain-controlled tension (consisting of a lattice with 30,648 bars, approximately $100 \times 100$ triangle cells) is given in Figure 3 . We take each breaking of bars as an individual event of microfracture. Initially, small events occur randomly and independently within the body. The interaction between such microfractures may be ignored. As a result, the macroscopic stress increases almost linearly at the initial stage, although quite a few randomly distributed microfractures have been observed. However, as the applied macroscopic deformation grows, especially in the vicinity of catastrophic rupture, the distribution of microdamages gradually evolves into another distinctive spatial pattern. The microfractures are tightly clustered in the space, and then form a major fault which finally causes catastrophic rupture. At this stage, the interaction between microfractures becomes significant. Individual small events are capable of cascading into much larger events and manifesting their effects on the macroscopic level. Such collective effects of microfractures are attributed to the dramatic redistribution of stress - complex nonlinear dynamics trigger catastrophic rupture. Thus, the emergence of macroscopically localized damage, which identifies a transition in the spatial pattern of microfractures, serves as a precursor of the catastrophic rupture in heterogeneous medium.

The criterion for damage localization discussed in the previous section provides a means for us to quantify the extent to which microfractures are grouped together, and thus serves as an important precursor of rupture. For example, the prediction of 
damage localization made by the mean-field approximation (the cross in Figure 3) is compared with observations of the damage pattern in Figure 3. The criterion provides an alarm prior to catastrophic failure. More importantly, the damage localization alarm is more sensitive than other clues of rupture. In fact, one cannot observe any sign of rupture in the damage patterns of Figure 3, even when the criterion provides an alarm. However, soon after the prediction, microfractures are observed to cluster and form a spatially localized damage distribution.

The influence of heterogeneity in microstructural strength is also examined in Figure 3. For a rather heterogeneous distribution in microstructural strength ( $m=2$, as shown in Figure 3a), the maximum macroscopic stress is lower than that for a relatively homogeneous distribution (for example, $m=5$, as shown in Figure 3b), while the emergence of damage localization is earlier than the latter one. This implies that unstable catastrophic ruptures seem more likely to occur at a low level of stress for a rather heterogeneous medium. It is also worth noting that microfractures in Figure $3 \mathrm{a}$ seem to distribute quite randomly in space. We can hardly predict the position where the final rupture appears, even when the medium is severely damaged. On the contrary, in Figure 3b, considerably less events can be detected before the catastrophic rupture. However, as it approaches the catastrophe transition point, microfractures are grouped together rapidly to form a major fault governing the final rupture. In spite of the differences in spatial evolution of damage, the criterion for damage localization provides a proper prediction for both cases.

In addition to its counterpart, the spatial pattern of damage, the temporal characteristics of energy release are examined in order to understand another essential aspect of the catastrophic rupture. Here, we load the network sample in such a way that the stress $\sigma$ is imposed and slowly increased, corresponding to a stress-controlled process under slowly increasing tectonic stress, as in seismicity. As shown in Figure 4, the thick line OABCDEFG represents a stress-strain curve for such a stress-controlled process. The energy release caused by bond breakage is related to each abrupt jump (A $\rightarrow B, C \rightarrow D$ or $\mathrm{E} \rightarrow \mathrm{F}$ in Figure 4 ) in the stressstrain curve. For instance, a jump from $\mathrm{C} \rightarrow \mathrm{D}$ at a stress level $\sigma_{C D}$ corresponds to an increment $\Delta \varepsilon$ in strain, then the released energy is $\Delta E=(1 / 2) \sigma_{C D} \cdot \Delta \varepsilon$, i.e., the area of OCD. Next, the temporal catalog of released energy is obtained as shown in Figure 5. The data indicates that the normalized energy release $\Delta E / \Delta E_{\max }\left(\Delta E_{\max }\right.$ denotes the maximum value of $\Delta E$ prior to the maximum stress) ranges from $10^{-5}-10^{0}$ for $m=2$, or $10^{-3}-10^{0}$ for $m=5$, spanning almost five orders in magnitude. The stress-strain curve is also provided in Figure 5 as a reference.

At the initial stage when the strain is between $[0,0.25]$ for $m=2$ or $[0,0.4]$ for $m=5$, the magnitude of energy release is always beneath $5.0 \times 10^{-3}$. The small energy release corresponds to individual microfractures during the globally stable stage. However, as the applied stress is slowly increased, the zone of influence of microfractures extends and may interact with each other. At this time avalanche coalescence becomes possible, and small events may cascade into much larger events. 


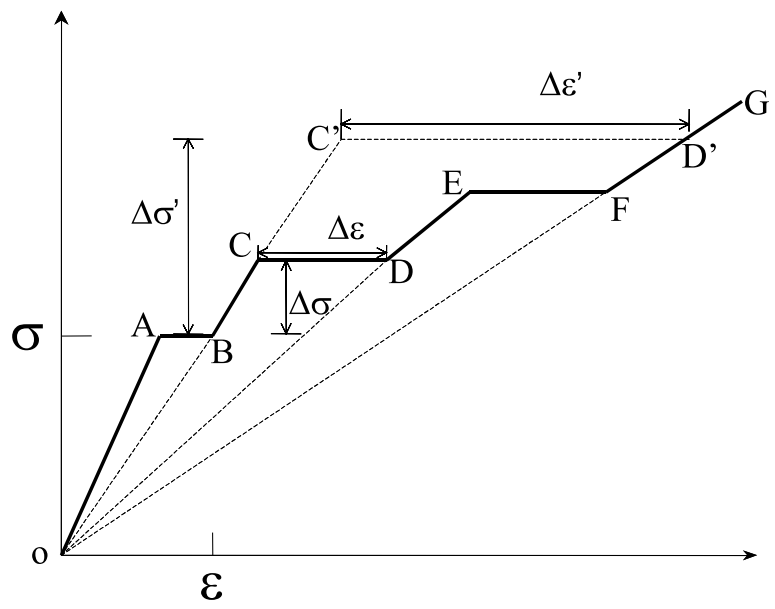

Figure 4

Schematic of the energy release for the present network model constrained by a stress-controlled process.

As a result, energy release of moderate magnitude $10^{-1}-10^{0}$ can be observed with increasing frequency and with higher amplitude until the final rupture occurs.

In particular, we compare the frequency-magnitude distribution of the first half of the energy release time-series in Figure $5\left(\varepsilon=0\right.$ to $\left.\varepsilon=\varepsilon_{f} / 2\right)$ to that of the second half of the sequence $\left(\varepsilon=\varepsilon_{f} / 2\right.$ to $\left.\varepsilon=\varepsilon_{f}\right)$, as shown in Figure 6. $\varepsilon_{f}$ represents the strain at which the main rupture initiates. It should be mentioned here that, for $m=5$, one can identify few events for the first half of the sequence. Thus, Figure $6 \mathrm{~b}$ plots only the distribution for the second half of the sequence. However, for both $m=2$ and $m=5$, the comparative plots indicate an overall change in the rate of energy release. Indicated by observations reported by JAUMÉ and SYKES (1999), there is evidence that some $M>6.5-7.0$ earthquakes are preceded by a period of increased occurrence of moderate (generally $M>5.0$ ) earthquakes in the region surrounding the oncoming large earthquake. Thus, the energy release time-series in Figure 5 exhibit a similar behavior in the activity of moderate events as shown in Figure 6.

Furthermore, we attempt to fit the cumulative energy release sequences to a power-law time to failure relation of the form:

$$
E(\varepsilon)=\sum_{\varepsilon_{t}=0}^{\varepsilon} \Delta E\left(\varepsilon_{t}\right)=A-B\left(\varepsilon_{f}-\varepsilon\right)^{n}, \quad n<1,
$$

where $A, B$ are empirical constants, and $n$ is the power-law exponent. In Figure 5 we overlay plots of the cumulative energy release versus strain with the plots of energy release time-series. $\varepsilon_{f}$ denotes the strain at which the main rupture initiates. For the stress-controlled process under consideration, $\varepsilon_{f}$ corresponds to the maximum stress. A nonlinear regression is then carried out to determine which group of values $(A, B, n)$ in Equation (11) minimizes the least-squares error of a 


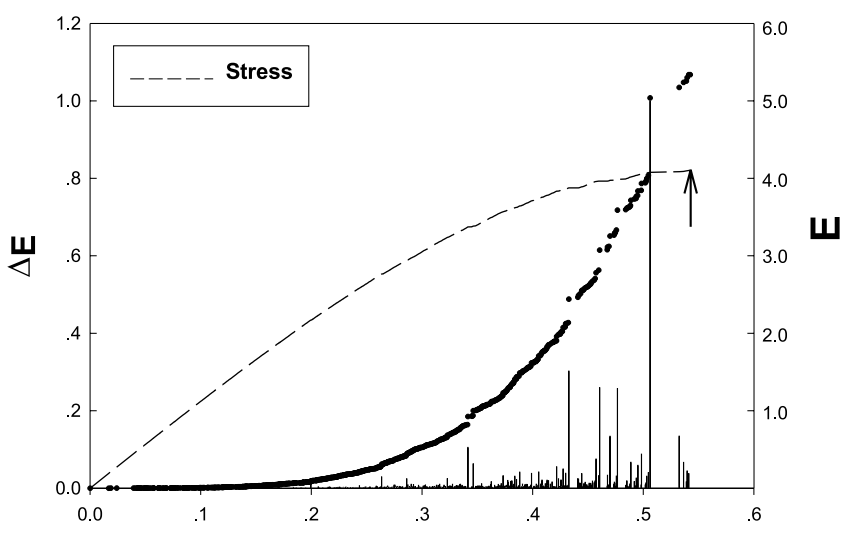

a)

$\varepsilon$

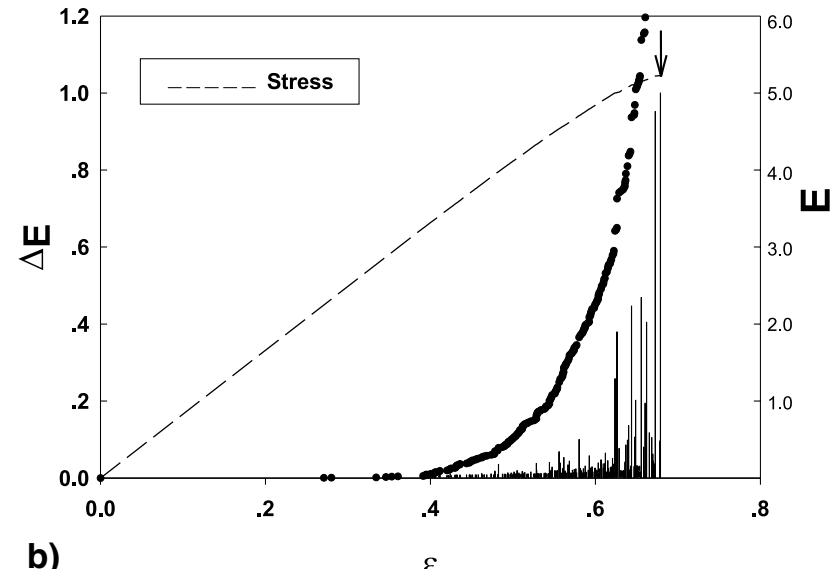

$\varepsilon$

Figure 5

The temporal pattern of the energy release together with the stress-strain curve for (a) $m=2$ and (b) $m=5$. Arrows ( $\uparrow$ for $m=2, \downarrow$ for $m=5$ ) on the stress-strain curves indicate the position where maximum stress appears. Circles - plots of the cumulative energy release versus strain.

power-law fit to the cumulative energy release data with the results shown in Figure 7 and Table 1.

In combination with the frequency-magnitude distribution mentioned above, the power-law fits to cumulative energy release provide a preliminary indication of accelerating energy release in the network model. This observational evidence preliminarily relates the catastrophe transition to critical point hypothesis $(\mathrm{CPH})$ of the earthquake. Thus, the network model may assist in quantifying the relationship between the two theories, and a statistical test is in progress to determine whether there is a systematic change in the rate of occurrence of events as the catastrophe transition is approached. 

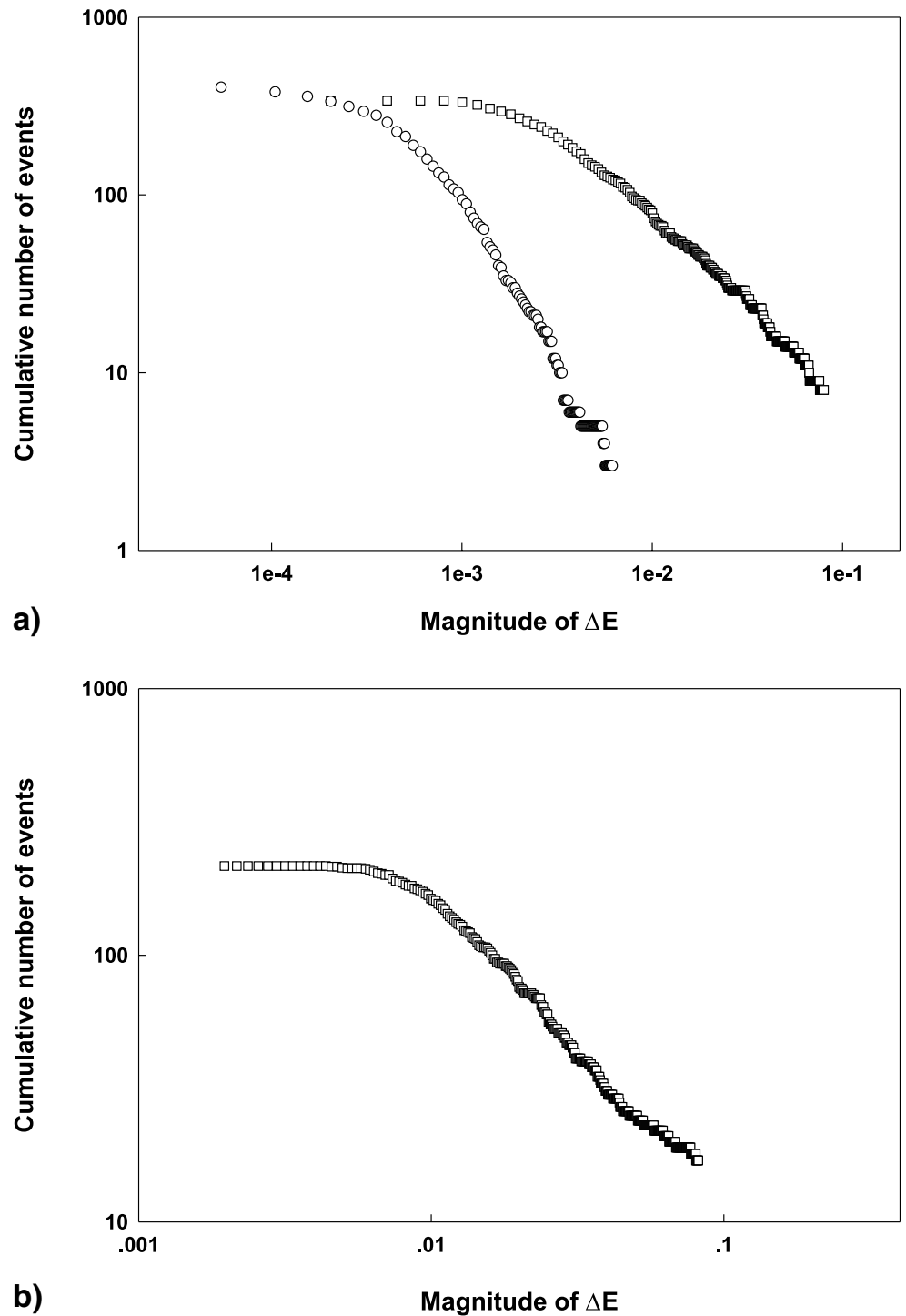

Figure 6

Comparative plots of the frequency-magnitude distribution of the first half of the energy release time-series ( $\varepsilon=0$ to $\varepsilon=\varepsilon_{f} / 2$, open circles) to that of the second half of the sequence $\left(\varepsilon=\varepsilon_{f} / 2\right.$ to $\varepsilon=\varepsilon_{f}$, open squares) (a) $m=2$ and (b) $m=5$. (Each abrupt jump in the stress-strain curve corresponds to an individual event in the energy release time-series. For $m=5$, few events can be observed for the first half of the sequence, thus the frequency-magnitude distribution is provided only for the second half of the sequence.)

In order to quantitatively describe the acceleration of energy release and extract its characteristics from the numerical data, the sensitivity of energy release to external stress $S=\left(\Delta E^{\prime} / \Delta \sigma^{\prime}\right) /(\Delta E / \Delta \sigma)$ is investigated, based on the numerical simulations 


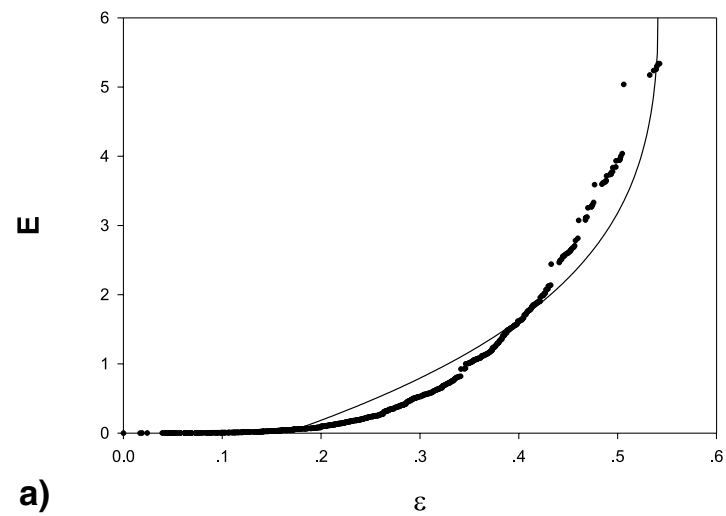

a)

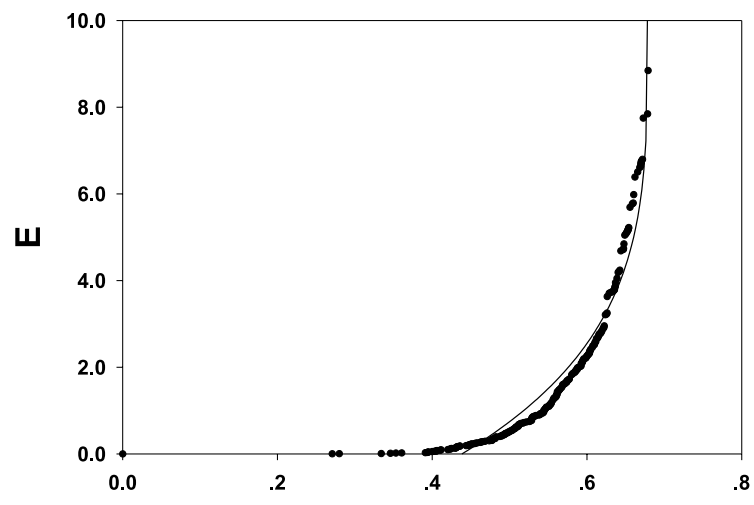

b)

$\varepsilon$

Figure 7

The power-law fits to cumulative energy release (a) $m=2$ and (b) $m=5$. Circles - plots of the cumulative energy release versus strain. Thin lines - curves of power-law fits.

(results are shown in Fig. 8). As shown in Figure 4, assume B corresponds to the initial state, an increment in external stress $\Delta \sigma(\mathrm{B} \rightarrow \mathrm{C} \rightarrow \mathrm{D})$ causes an abrupt jump, and the corresponding energy release $\Delta E=1 / 2(\sigma+\Delta \sigma) \cdot \Delta \varepsilon$. Meanwhile, as a virtual increment of stress, $\Delta \sigma^{\prime}$ is imposed almost immediately $\left(\mathrm{B} \rightarrow \mathrm{C}^{\prime} \rightarrow \mathrm{D}^{\prime}\right)$ and it takes a short enough time in comparison to the time-scale associated with the slowly increasing tectonic loading. $\Delta \varepsilon^{\prime}$ is the corresponding virtual jump in strain. ( $\Delta \sigma^{\prime}=\Delta \sigma+\alpha \Delta \sigma_{0}$, where $\alpha$ is a trial parameter and $\Delta \sigma_{0}$ is a characteristic increment of the same order as $\Delta \sigma . \alpha \Delta \sigma_{0}$ is relatively small compared to the magnitude of $\sigma$ itself.) Under the small virtual increment of loading $\Delta \sigma^{\prime}$, the energy release will be $\Delta \mathrm{E}^{\prime}=1 / 2\left(\sigma+\Delta \sigma^{\prime}\right) \Delta \varepsilon^{\prime}$ i.e., the area of $\mathrm{OC}^{\prime} \mathrm{D}^{\prime}$.

XIA et al. (2000) have shown that sensitivity increases significantly prior to the catastrophe transition from the globally stable (GS) stage to the main rupture, via evolution-induced catastrophe (EIC) in the nonlinear model. This feature is called 
Table 1

Parameters obtained by the power-law fits to cumulative energy release

\begin{tabular}{ccc}
\hline & Coefficient & Std. Error \\
\hline (a) $m=2, \varepsilon_{f}=0.5423$ & & \\
$\mathrm{~A}$ & 7.5764 & 0.2430 \\
$\mathrm{~B}$ & 9.6482 & 0.2204 \\
$\mathrm{n}$ & 0.2486 & 0.0104 \\
& & \\
(b) $m=5, \varepsilon_{f}=0.6789$ & 11.0213 & 0.4455 \\
$\mathrm{~A}$ & 15.4274 & 0.3813 \\
$\mathrm{~B}$ & 0.2359 & 0.0135 \\
$\mathrm{n}$ & & \\
\hline
\end{tabular}

critical sensitivity. Here, for a computational approximation of a two-dimensional heterogeneous medium, the critical sensitivity of energy release to external stress manifests itself prior to the catastrophe transition, as shown in Figure 8. $S \sim 1$ means that minor variation in governing parameters would not trigger any exaggerated consequences; there are independent random events only. While, if $S>1$, it implies that the system has become sensitive and minor variations in governing parameters may induce multi-scale coalescence. In Figure 8, as the external loading is slowly increased, a significant increase in the sensitivity can be observed for both $m=2$ and $m=5$. Increasing sensitivity indicates that small events have a considerably higher probability of growing into a larger event, which may lead to final rupture. Actually, the critical sensitivity is essentially rooted in the nonlinear dynamics of the transition from globally stable accumulation to catastrophic rupture. Thus, it provides an inherent precursor by quantitatively measuring the response of energy release to external loading.

When the maximum stress is attained, the catastrophe transition becomes possible, as discussed in section 2. Unfortunately, it is difficult for the present network model to simulate the catastrophe transition process beyond maximum stress precisely. The damage localization likely may lead to a large local deformation in the vicinity of major fault. The network model fails to properly describe extremely large local deformation. Therefore, in this paper, we report the simulations of energy release until maximum stress only. Precise numerical investigation beyond this point has met with some technical difficulties and further research continuous.

\section{Conclusions}

Both damage localization and sensitivity of energy release result from the cascade of microdamage coalescence. Damage localization is the spatial representation of damage evolution, whereas the sensitivity of energy release is its temporal counterpart. The former can be examined in terms of a smoothly fitted dynamic function of damage or a mean-field approximation. The latter can be calculated 


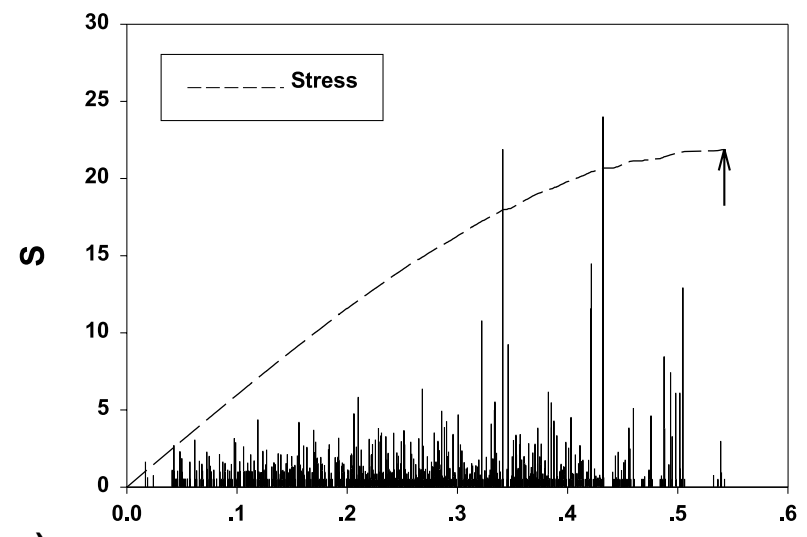

a)

$\varepsilon$

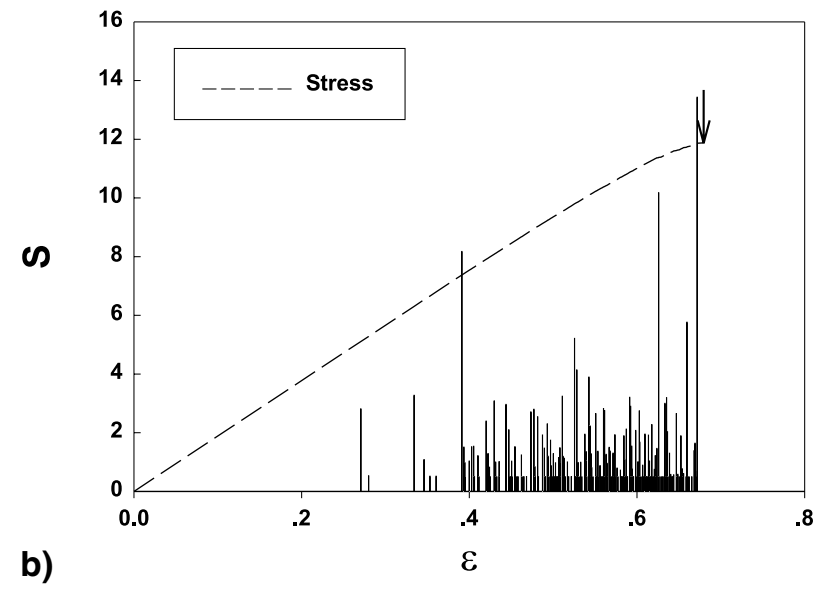

Figure 8

The sensitivity of energy release versus strain in a heterogeneous network model possessing a Weibull distribution function with (a) $m=2$ and (b) $m=5\left(\alpha=1.0, \Delta \sigma_{0}=\Delta \sigma\right.$ ). Arrows ( $\uparrow$ for $m=2$, $\downarrow$ for $m=5$ ) on the stress-strain curves indicate the maximum stress. Notice the rapid increase in sensitivity when approaching the maximum stress.

directly in the light of discrete intermediate-sized events. From the simulations, it can be seen that both damage localization and critical sensitivity of energy release can provide an alarm prior to an impending rupture. However, for accurate prediction of a rupture, there is still need to examine the relationship between various precursors and the main rupture, especially those beyond the maximum stress.

The above power-law fits show that accelerating energy release precedes the catastrophe transition. This is an indication that the catastrophe transition and critical point hypothesis $(\mathrm{CPH})$ of an earthquake are closely interrelated. The two theories may highlight complementary aspects of the physics associated with catastrophic failure in general and earthquake forecasting in particular. 


\section{Acknowledgments}

This research was funded by the Special Funds for Major State Basic Research Projects (G2000077305) and the National Natural Science Foundation of China (19891180,19732060, 19972004, 10002020 and 10047006).

\section{REFERENCES}

BAI, Y. L., BAI, J., LI, H. L., KE, F. J., and XIA, M. F. (2000a), Damage Evolution Localization and Failure of Solid Subjected to Impact Loading, Int. J. Impact Eng. 24, 685-701.

BAI, Y. L., WeI, Y. J., XIA, M. F., and Ke, F. J. (2000b), Weibull Modulus of Diverse Strength due to Sample-specificity, Theor. Appl. Fract. Mech. 34, 211-216.

BAI, Y. L., XIA, M. F., Ke, F. J., and LI, H. L., Damage field equation and criterion for damage localization. In Rheology of Bodies with Defects, Proc. of IUTAM Symposium (ed. R. Wang) (Kluwer Academic Publishers, Dordrecht 1998) pp. 55-66.

JAEger, J. C. and COOK, N. G., Fundamentals of Rock Mechanics (Chapman and Hall, London 1979) p. 461.

Jaumé, S. C. and Sykes, L. R. (1999), Evolving Towards a Critical Point: A Review of Accelerating Seismic Moment/Energy Release Prior to Large and Great Earthquakes, Pure Appl. Geophys. 155, 279-306.

Krajcinovic, D., Damage Mechanics (Elsevier Science B.V., Amsterdam 1996).

LI, H. L., BAI, Y. L., XIA, M. F., and Ke, F. J. (2000), Damage Localization as a Possible Precursor of Earthquake Rupture, Pure Appl. Geophys. 157, 1945-1957.

Liu, X. Y., Yan, W. D., and Liang, N. G. (1998), A Pseudo-plastic Engagement Effect on the Toughening of Discontinuous Fiber-reinforced Brittle Composites, Metals And Materials 4, 242-246.

Lu, C., Vere-Jones, D., and Takayasu H. (1999), Avalanche Behavior and Statistical Properties in a Microcrack Coalescence Process, Physical Review Letters 82, 347-350.

Rundle, J. B., Klein, W., Turcotte, D. L., and Malamud, D. (2000), Precursory Seismic Activation and Critical Point Phenomena, Pure Appl. Geophys. 157, 2165-2182.

Sammis, C. G., Sornette, D., and Saleur, H., Complexity and earthquake forecasting. In Reduction and Predictability of Natural Disasters (eds. Rundle, J. B., Turcotte, D. L., and Klein, W.) (Addison Wesley 1994) pp. 143-156.

XIA, M. F., WeI, Y. J., BAI, J., Ke, F. J., and BAI, Y. L. (2000), Evolution Induced Catastrophe in a Nonlinear Dynamical Model of Material Failure, Nonlinear Dynamics 22, 205-224.

XIA, M. F., WEI, Y. J., KE, F. J., and BAI, Y. L., Critical Sensitivity and Fluctuations in Catastrophic Rupture. In 2-nd ACES Workshop (The APEC Cooperation for Earthquake Simulation, 2001) pp. 125-130.

XIA, M. F., Ke, F. J., BAI, J., and BAI, Y. L. (1997), Threshold Diversity and Trans-Scales Sensitivity in a Finite Nonlinear Evolution Model of Materials Failure, Phys. Lett. A 236, 60-64.

XIA, M. F., Song, Z. Q., Xu, J. B., ZhaO, K. H., and BAI, Y. L. (1996), Sample-Specific Behavior in Failure Models of Disordered Media, Commun. Theor. Phys. 25, 49-54.

(Received February 21, 2001, revised June 11, 2001, accepted June 25, 2001)

To access this journal online:
hiftp://www.birkhauser.ch 